\title{
Kurze Mittheilung über Anwendung der Salicylsäure.
}

\author{
Von \\ C $\mathbf{r} \theta \mathbf{d} \dot{\theta}$.
}

Auf Empfehlung des Herrn Geh. Hofrathes Kolbe wird die Salicylsäure seit Juli $d$. J., wie in der chirurgischen, so auch in der hiesigen gynäkologischen Klinik fast ausschliesslich an Stelle der Carbolsäure verwendet, zur Desinfection der Hände, Vaginaldouchen der Wöchnerinnen, Bestreuen der Ulcera puerperalia u. s. W., und zwar in Lösungen von 1:300 bis 1:900, oder als Pulver gemischt mit Amylum 1:5 oder in Form der salicylsauren Watte. Die Anwendung des Mittels hat bis jetzt so günstige Erfolge gezeigt, dass dasselbe jetzt schon allgemein für geburtshülfliche Praxis dringend empfohlen werden soll; ein ausführlicherer Bericht darüber wird erst später erfolgen.

Leipzig, December 1874.

\section{Zur Genese der Amnionzotten.}

Antwort auf die Erwiderung des Herrn Dr. F. N. Winkler in Dresden (Archiv, Band VII, Seite 325). ${ }^{1}$ )

Yon

Dr. Ahlfeld.

Ich halte die Unterschiede zwischen den Befunden physiologischer Carnnkeln des Amnion und den von mir beschriebenen Zotten nicht so gross, dass ich glaube, sie genetisch trennen zu müssen. Auf den ersten Anblick freilich lassen sich die Bilder 8 und 22 anf Tafel I der Winkler'schen Abhandlung nicht gut vereinen. Wenn auch Winkler für seine Person unter Carunkel ein reines Epithelialgebilde verstebt, so stehen ihm doch andere Autoren entgegen, welche den Begriff der Carunkel anders definiren. Cl. Bernard sagt, dass

1) Mit dieser Antwort betrachtet die Red. die Sache für abgeschlossen.

Archiv f. Gyuäkologie. Bd, VII. Hft. 3. 
die Carunkeln bei Wiederkäuern in gewissen Bild ungsstadien a f flachen Hugeln des amniotischen Bindegewebes aufsitzen. Mikroskopisch fand auch Dohrn, wie ich in meinem Aufsatze erwähnte, den Cebergang von platten Carunkeln zu gestielten Papillen. Er schreibt: Sie zeigen zuweilen eine deutlich papilläre Form, in anderen Fälen findet man nur halbkugelige Prominenzen oder ganz unregelmässig geformte Erhebungen der Oberfläche.

Die Papillenform durch einfache Schichtung der Epithelien entstanden zu denken, ist mir unmöglich. Es muss die Papille einen Bindegewebsstock haben, sonst würde das Epithel maceriren and $a b$ fallen; um so mehr, da sie im Fruchtwasser schwimmt. Betheiligt sich aber in der That die Bindegewebsschicht an der Bildung der Carunkel, so liegt nichts näher, als auch der Gallertschicht die Möglichkeit, zugestehen zu müssen, in die Ausbuchtnng der Bindegewebsschichte hineinzuwuchern und das Innere der Carunkel oder Zotte auszufitlen. Ist auf diese Weise eine Papille oder Zotte entstanden, und dieselbe ist macerirt, so schwindet das Epithel, und es bleibt die macerirte Bindegewebs und Gallertschicht übrig, die dann sehr wohl ein Bild wie in Fig. 8. bei Winkler abgeben können.

So habe ich mir meine Zotten aus der Carunkel entstanden gedacht. Unterstützt wird diese Anschauung durch die Thatsache, dass in dem Falle 2, Seite 363 meiner Arbeit, von einex auffallend dicken Gallertschicht die Rede ist; unterstutzt wird sie fernex durch die eigenthümliche Vertheilung von Carunkeln und Zotten im Falle 1 , indem beide streifenweise neben einander anf dem Amnion au finden waren. Ich erkläre mir dies so, dass die frei in die Amnionhöhle hineinragenden Carunkeln, durch den Niederschlag aus dern Fruchtwasser bedeckt, an weiterem Wachsthume gehindert wurden, wäluend die in Falten des Amuion liegenden unbehelligt weiter wuchern. kounten.

Ich war so fest davon uberzeugt, dass die ron Winkleige. fundenen Zotten mit den meinen identisch seien, dass ich ars diesem Grunde zwei Beobachtungen Winkler's füir irrthümlich hielt:

Erstens habe ich irrthimlicher Weise an die Möglichkeit ge. dacht, dass die feineren Zeichnungen anf der Zotte (Fig. 8 bei Winkler) Reste von Epithelien sein könnten, obgleich Winkler angiebt, die betreffenden Zotten hätten keine zelligen Bestandtheile gezeigt. Mein Irrthum ist abex insofem in etwas verzeihlich, als man nicht erwarten konnte, dass durch jene dicke Zotte, wenn sie auch pellucid war, die darunter liegenden Epithelien zu schen waren.

Zweitens habe ich nicht geglaubt, dass Wink ${ }^{r}$ er ernstich der Intercellularsubstanz des Bindegewebes eine Rolle suertheilt, Tropfen zu bilden, die exstarien und frei in die Amnionhoble hineinragen sollen. Es liegt diese Anschaung soweit ansserhalb des Bereiches aller bisher bekannten histologischen Thatsachen, dass ich vermuthete, Winkler habe die Gallertschieht gemeint. Wurde abex die Haupt- 
masse der Zotte von der Gallertschicht geliefert, was ich auch jetzt noch nicht bezweifele, so muss sie Winkler, da Bindegewebsschicht und Epithelschicht fehlten, als nicht vom Amnion ausgehend erklären, während ich sie als Amnionzotten bezeichnete, deren Epithel- und Bindegewebsschicht nicht mehr nachweisbar sind. Auf jeden Fall aber ist das Unrecht auf"meiner Seite, dass ich, entgegen Winkler's Angaben, ihm meine Ansichten subponirt habe.

Wenn Winkler in der Erwiderung, Seite 325, sagt: „Das Gewebe dieser Keulen glich durchaus dem des Stratum," so genügt eine Durchsicht der von ihm gegebenen Beschreibung der Keulen, um ziI constatiren, dass dies nicht der Fall war, dass vielmehr die Masse vollständig homogen war und ganz den Eindruck „der Intercellularsubstanz des Substrates" machte, aber nicht den Eindruck des Stratums.

Gewebe ist ein Ausdruck für eine differenzirte, aus Zellen bestehende Bildung. Da Winkler in seiner Beschreibung Zellen ausdrücklich negirt, so darf er jetzt nicht von einem "Gewebe" seiner Keulen reden.

Unerklärlich bleibt es mir, wie Winkler, während er mir den Vorwurf macht, dass ich ihm ,ohne Weiteres einen recht groben Beobachtungsfehler imputirt" habe, er auf derselben Seite sich des gleichen Vergehens schuldig macht, dessen er mich zeiht.

Obgleich ich in meiner Arbeit deutlich gesagt habe: „F'ür meine Zotten lässt sich aber nachweisen, dass sie wahre Amnionzotten, wenigstens mit Amnionepithel bekleidet sind (Seite 359); es ist mir geglicket, Zotten von Amnion zu trennen, und auch diese liessen die epithelartige Zeichnung erkennen (Seite 361); da ich nun an anderen Stellen des Präparates deutliche Epithelien a uf der Zotte gesehen habe, ja in einigen Fällen den Uebergang derselben vom Amnion auf die Zotte nach. weisen konnte (Seite 361); nur in wenigen Fällen war es nachweisbar, dass dieses Epithel von der Amnionfläche chne Unterbrechung a uf den Stiel und die Zotte übergeht. Doch konnte durch einige Bilder dieser Uebergang sichergestellt werden (Seite 361) r. s. W. u. s. w., schreibt Winkler (Seite 327 im Archiv): Ich habe jedoch Gründe, die Ahlfeld'sche Behauptung von der Existenz dieses Epithels nicht ohne Weiteres zu acceptiren, und Seite 329: Unter diesen Umständen wird man wohl die Existenz einer Epitheldecke auf diesen Excrescenzen vorläufig noch als eine offene Frage behandeln müssen.

Die Gründe für diese Behauptung gièbt $W$ inkler Seite 328 und 329 an. Es ist mir sehr leicht, dieselben zu widerlegen:

Winkler behauptet, ich hätte die Zotten nur an meinem ersten Präparate untersucht, welches zu Untersuchungen der Art nicht geeignet gewesen sei. Er befindet sich im Irrthume. Seite 361, unten, habe ich deutlich geschrieben, wie an der frischen zweiten Placenta ebenfalls die mikroskopische Untersuchang angestellt worden ist. 
Ferner führt Winkler als zweiten Grund den an, dass sich bej mir nicht die leiseste Andeutung von Anwendung von Reagentien erwähnt finde. Es handelt sich in unserem Falle um den Nachweis des Vorhandenseins von Epithelien auf den Zotten. Sind diese Epithelien zu sehen, mag das Präparat noch so schlecht behandelt worden sein, so ist der Beweis ihrer Anwesenheit geliefert.

Drittens glaubt Winkler an der Existenz des Epithels zweifeln zu duirfen, weil in der Abbildung, Fig. 8 meiner Tafel, sich nur undeutlich die Conturen von zwei Epithelzellen gezeichnet finden. Winkler übertreibt, wenn er sagt, man sehe auf den Excrescenzen nichts.

Die Zeichnungen auf Tafel 5 sind sämmtlich nach der Natur gezeichnet. Herr Dr. Thierfelder, einer der geübtesten Zeichner mikroskopischer Präparate, hat die Guite gehabt, sie mir auszuführen. In seiner Zeichnung waren die Conturen der Zellen ein wenig schärfer ansgedrückt. Jeder, wer mit derartigen feinen Arbeiten zu thun hat, weiss, dass auf den Druck der Lithographie sehr viel ankommt. Hier ist er ein wenig zu schwach gewesen, daher die Conturen nicht so dentlich, als Herr Dr. Thierfelder und ich gewünscht hatten.

Im iibrigen giebt eine misslungene Abbildung keine Berechtigung, an der Wabrheit der im Texte so bäufig und deutlich ausgesprochenen Befonde zweifeln zu dürfen.

Dass Herr Dr. Thierfelder gerade das Präparat, was in Fig. 8 abgebildet ist, mir zeichnete, hat seinen Grund darin, dass er zufullig in Besitze desselben war, als ich ihn um eine Zeichnung bat. Unserer Ansicht nach war dies Präparat ebenso beweisend, wie eins mit einer grösseren Anzahl Epithelien.

Was nun die Behandlung meiner Prüparate anbetrifft, so ist diese, ausser von mir, auch von Hern Dr. Thierfelder, erstem Assistenten des hiesigen pathologischen Institntes, ausgefiihrt worden. Zum Theil sind die Bilder gefürbt, die Firbung gut gelungen.

Von dem Vorhandensein des Epithels auf der Zotte und von dem Uebergange des Epithels von Amnion auf Zotte, baben sich die Herren Professoren Wagner, Schüppel in Tübingen, Dr. Thierfelder und eine Anzahl Collegen iberzeugt.

In neuester Zeit ist bereits eine Bestätigung meiner Beschreibungen und Ansichten veröffentlicht woxden. Herr Dr. Leopold hat der geburtshülflichen Gesellschaft zu Leipzig Zotten vorgelegt, deren Bau, da es ein ganz frisches Präparat, sehr gut zu demonstriren war.

Herr Leopold zeigte Präparate, in denen der Uebergang won der einfachen Carunkel bis zur Zotte gut, zu sehen war. Eine kurge Beschreibung ${ }^{1}$ ) dieses Befundes befindet sich im Archive, oben S. 389.

1) In dem betreffenden Referate ist ein entstellender Fehler za corrigiren, indem statt "Injection 